Pacific Journal of Mathematics

SEMI-SIMPLE RADICAL CLASSES OF ALGEBRAS AND be ry. GR D 


\title{
SEMI-SIMPLE RADICAL CLASSES OF ALGEBRAS AND ATTAINABILITY OF IDENTITIES
}

\author{
B. J. GARDNER
}

This paper is concerned with the investigation of some interplay between the theories of (Kurosh-Amitsur) radicals and varieties of (not necessarily associative) algebras. Specifically, it is shown that a variety is a radical class if and only if it is closed under extensions, while a radical class which is also a semi-simple class is the same thing as a variety with attainable identities in the sense of T. Tamura (J. Algebra 3 (1966), 261-276). In certain instances it is shown that the two properties of varieties are equivalent.

The semi-simple radical classes of associative rings have been described by P. N. Stewart and have subsequently been investigated by several authors. The necessary modifications of Stewart's work are carried out in $\S 2$ of the present paper to provide a classification of the semi-simple radical classes of associative algebras over an arbitrary field. It turns out that if the field is infinite there are only the trivial classes, while for finite fields the classes are the appropriate portions of the semi-simple radical classes of rings. In $\S 3$ some examples of the presence and absence of nontrivial semisimple radical classes in non-associative situations are given.

Tamura introduced the notion of attainability while dealing with semigroups (and thus his formulation differs somewhat from the special case relevant to our considerations, which we introduce in $\S 1$ ) and posed the problem of finding all attainable sets of identities for rings and other structures. The matter was taken up by Mal'tsev [18], who showed that. (in situations where the concept is defined) if a variety has attainable identities, it is closed under extensions. Whether the converse is true or not appears to be an open question. In this paper we show that the converse is true in a number of cases, including associative, alternative and Jordan (if the operator domain contains 1/2) algebras over a principal ideal domain-in these cases giving at least some examples of varieties with the properties. In some other cases, including arbitrary and Lie algebras over fields and the ring of integers, we show that there are no nontrivial varieties with attainable identities, so the problem here is to determine whether or not there are nontrivial varieties closed under extensions. In the case of Lie algebras over fields of characteristic 0, Parfenov [22] has shown that there aren't any.

Our references for variety theory are the long article of Osborn 
[21] and the book by Procesi [23]. The latter treats only associative algebras. For radical theory see Divinsky [5], or the notes of Leavitt [14] or Wiegandt [35]. Leavitt deals with non-associative rings, the others with associative rings only.

It is customary to state that radical theory can be developed in any "universal class" of rings or algebras-a class closed under ideals and homomorphic images. All the universal classes in which we shall work will be varieties, which we designate universal varieties. Two other conventions used throughout the paper are worth mentioning: any class of algebras is assumed to contain, along with each member, all isomorphic copies thereof; $\triangleleft$ means "is an ideal of".

1. General results. Throughout this section we shall work in a fixed "universal" variety $\mathscr{W}$ of algebras over a commutative associative ring $\Omega$, with an identity element. All varieties treated will be "within" $\mathscr{W}$.

Let $F$ denote a free algebra (in $\mathscr{W}$ ) on a countably infinite set of generators. A $T$-ideal of $F$ is an ideal which is invariant under every endomorphism of $F$. Let $I$ be a $T$-ideal of $F$. For each algebra $A$, we denote by $A(I)$ the subalgebra of $A$ generated by

$$
\bigcup\{f(I) \mid F \stackrel{f}{\longrightarrow} A\} \text {. }
$$

Then $A(I)$ is the set of evaluations in $A$ of polynomials in $I$. There is a bijection between the set of $T$-ideals of $F$ and the set of varieties, given by associating with each $T$-ideal $I$ the class $\mathscr{T}$ of algebras in which the polynomials from $I$ vanish identically. Moreover, for every algebra $A$, we have

$$
A / A(I) \in \mathscr{C} \quad \text { and } \quad A(I)=\bigcap\{K \triangleleft A \mid A / K \in \mathscr{C}\} .
$$

In addition, $\mathscr{V}=\{B / B(I) \mid B \in \mathscr{W}\}$.

Being hereditary, a variety of defines an upper radical class $U(\mathscr{V})$. Our first result relates this class to the concepts discussed above.

THEOREM 1.1. Let of be a variety, I the associated T-ideal. Then

$$
\{A \mid A(I)=A\}=U(\mathscr{Y})
$$

Proof. The upper radical class consists of those algebras with no nonzero homomorphic images in $\mathscr{V}$. By our remarks above, these are precisely the algebras $A$ with $A(I)=A$.

Shchukin [24] has shown that in the class of all groups, for any 
fixed variety, the class of groups coinciding with their verbal subgroups (relative to this variety) form a radical class. The group theory analogue of Theorem 1.1 (and its proof) provide another way of getting Shchukin's result.

In the sequel we shall denote the radical class of Theorem 1.1 by $\mathscr{R}_{I}$.

In some cases we can get a "local" characterization of $\mathscr{R}_{I}$, using a transfinite chain of subalgebras defined as follows ( $I$ is a $T$-ideal): $A(I, 1)=A(I) ; A(I, \alpha+1)=A(I, \alpha)(I)$ for all ordinals $\alpha ; A(I, \beta)=$ $\bigcap_{\alpha<\beta} A(I, \alpha)$ if $\beta$ is a limit ordinal. For each algebra $A$, the chain

$$
A \supseteq A(I, 1) \supseteqq A(I, 2) \supseteqq \cdots \supseteqq A(I, \alpha) \supseteqq A(I, \alpha+1) \supseteqq \cdots
$$

must terminate. If $A(I, \mu)=A(I, \mu+1)$, we can write $A(I, \mu)=$ $\bigcap_{\alpha} A(I, \alpha)$. Note that $\bigcap_{\alpha} A(I, \alpha) \in \mathscr{R}_{I}$.

Recall that a radical class is strict if every radical subalgebra of every algebra is contained in the radical or, equivalently, if subalgebras of semi-simple algebras are semi-simple.

THEOREM 1.2. The following conditions are equivalent for a T-ideal $I$ :

(i) $\mathscr{R}_{I}$ is strict;

(ii) $\mathscr{R}_{I}(A)=\bigcap_{\alpha} A(I, \alpha)$ for all algebras $A$.

Proof. (i) $\Rightarrow$ (ii): As we have observed, $\bigcap_{\alpha} A(I, \alpha)$ is in $\mathscr{R}_{I}$ for each algebra $A$, so, $\mathscr{R}_{I}$ being strict, $\bigcap_{\alpha} A(I, \alpha) \subseteq \mathscr{R}_{I}(A)$. On the other hand, it is clear that $\mathscr{R}_{I}(A)=\mathscr{R}_{I}(A)(I) \cong A(I)=A(I, 1)$. If $\mathscr{R}_{I}(A) \subseteq A(I, \alpha)$ then

$$
\mathscr{R}_{I}(A)=\mathscr{R}_{I}(A)(I) \cong A(I, \alpha)(I)=A(I, \alpha+1),
$$

while if $\mathscr{R}_{I}(A) \subseteq A(I, \alpha)$ for all $\alpha<$ a limit ordinal $\beta$, then $\mathscr{R}_{I}(A) \subseteq$ $A(I, \beta)=\bigcap_{\alpha<\beta} A(I, \alpha)$. Hence $\mathscr{R}_{I}(A) \leqq \bigcap_{\alpha} A(I, \alpha)$.

(ii) $\Rightarrow$ (i): If $B$ is a subalgebra of an algebra $C$, then clearly $B(I) \subseteq C(I)$ so a simple induction argument shows that $B(I, \alpha) \subseteq$ $C(I, \alpha)$ for each $\alpha$. In particular, if $B$ is in $\mathscr{R}_{I}$, then $B=\mathscr{R}_{I}(B)=$ $\bigcap_{\alpha} B(I, \alpha) \subseteq \bigcap_{\alpha} C(I, \alpha)=\mathscr{R}_{I}(C)$.

CoROLlary 1.3. If $\mathscr{W}$ is the variety of all associative rings, then for every $T$-ideal $I, \mathscr{R}_{I}(A)=\bigcap_{\alpha} A(I, \alpha)$ for every ring $A$.

Proof. By Theorem 2.5 of [8], for any variety $\mathscr{V}$ of associative rings we have 

$U(\mathscr{V})=\{A \mid A$ has no nonzero homomorphisms with accessible images to rings in $\mathscr{V}\}=\{A \mid A$ has no nonzero homo- morphisms to rings in $\mathscr{V}\}$.

(The latter equality follows from the fact that $\mathscr{V}$ is strongly hereditary, i.e., closed under formation of subrings.) By Proposition 3.6 of [8] (taking for $\Sigma$ the property of being a subring) $U(\mathscr{Y})$ is strict.

Corollary 1.3 cannot be generalized without restriction. When $\mathscr{W}$ is the variety of all rings and $I$ the $T$-ideal generated by $x y$, then $\mathscr{R}_{I}$ is the class of idempotent rings and its semi-simple class is not even hereditary [16].

The product $\mathscr{X} \circ \mathscr{Y}$ of two varieties $\mathscr{X}$ and $\mathscr{Y}$ is the class of algebras $W$ for which there exists a short exact sequence

$$
0 \longrightarrow X \longrightarrow W \longrightarrow Y \longrightarrow 0
$$

with $X \in \mathscr{X}$ and $Y \in \mathscr{Y}$. Naturally, we call a variety $\mathscr{X}$ idempotent if $\mathscr{X} \circ \mathscr{X}=\mathscr{X}$.

THEOREM 1.4. The following conditions are equivalent for a class of of algebras:

(i) Or is a radical class which is closed under formation of direct products and subalgebras;

(ii) $\mathscr{V}$ is an idempotent variety.

Proof. Clearly (i) $\Rightarrow$ (ii).

(ii) $\Rightarrow$ (i): If $\mathscr{V}$ is an idempotent variety, it is closed under homomorphic images and extensions. It is also closed under subdirect products and hence is local, i.e. unions of directed systems of subalgebras from $\mathscr{V}$ are in $\mathscr{Y}$. (See [4], p. 101; also [35], p. 161.) In particular, unions of chains of $\mathscr{V}$-ideals are $\mathscr{V}$-ideals. It follows that $\mathscr{V}$ is a radical class. Being a variety, $\mathscr{C}$ has the other required closure properties.

The identities of a variety of with $T$-ideal $I$ are said to be attainable if $A(I, 2)=A(I, 1)$ for all algebras $A$. The concept of attainability was introduced by Tamura [33] for semigroups and discussed also by Mal'tsev [18].

THEOREM 1.5. The following conditions are equivalent for a class $\mathscr{V}$ of algebras:

(i) y is a variety with attainable identities;

(ii) $\mathscr{V}$ is a homomorphically closed semi-simple class;

(iii) $\mathscr{V}$ is a semi-simple radical class.

Proof. (i) $\Rightarrow$ (ii): Let $I$ be the $T$-ideal associated with $\mathscr{Y}, \mathscr{S}$ the 
semi-simple class associated with $\mathscr{R}_{I}$. Then for each algebra $A$, we have $A(I)=A(I, 1)=A(I, 2)=A(I)(I)$, so $A(I) \in \mathscr{R}_{I}$, while $A / A(I) \in$ $\mathscr{V} \cong \mathscr{S}$. It follows that $A(I)=\mathscr{R}_{I}(A)$ for all algebras $A$. Hence

$$
\mathscr{V}=\{A|A(I)| A \in \mathscr{W}\}=\left\{A\left|\mathscr{R}_{I}(A)\right| A \in \mathscr{W}\right\}=\mathscr{S}
$$

so $\mathscr{V}$ is a semi-simple class, clearly homomorphically closed.

(ii) $\Rightarrow$ (iii): $\quad \mathscr{V}$ is closed under homomorphic images and subdirect products and hence is strongly hereditary, by an argument identical to that used in [31], Lemma 4.1 and [35], p. 163. $\mathscr{V}$ is therefore an idempotent variety and so, by Theorem 1.4, a radical class.

(iii) $\Rightarrow$ (i): Since $\mathscr{V}$ is closed under subdirect products and homomorphic images, it is strongly hereditary, and hence a variety as well as a semi-simple class. Let $I$ be the $T$-ideal associated with $\mathscr{V}$ and let $\mathscr{\mathscr { C }}=U(\mathscr{V})$. Then for every algebra $A$, we have

$$
A(I)=\bigcap\{K \triangleleft A \mid A / K \in \mathscr{V}\}=\mathscr{U}(A),
$$

so $A(I, 2)=A(I)(I)=\mathscr{U}(\mathscr{C})(A)=\mathscr{U}(A)=A(I)=A(I, 1)$, i.e. $\mathscr{V}$ has attainable identities.

CoRollaRY 1.6 (Mal'tsev [18]). If a variety gr has attainable identities, then of is idempotent.

We do not know of an idempotent variety which has nonattainable identities. Wiegandt [35] has shown that for associative rings, a semi-simple radical class is the same thing as an idempotent variety. We shall consider some further instances of this phenomenon. For this we place some restrictions on our universal variety $\mathscr{W}$.

We first assume that $\mathscr{W}$ contains all the zeroalgebras over $\Omega$, i.e. the algebras with trivial multiplication.

We shall call an algebra $A \in \mathscr{W}$ nilpotent of index $n$ if every product of $n$ elements of $A$, with arbitrary bracketing, is zero, but there exists a nonzero product of $n-1$ elements. A product $b$ of the latter kind satisfies the conditions

$$
b A=0 ; A b=0 \text {. }
$$

Thus the ideal generated by $b$ is a zeroalgebra. Since the class of nilpotent algebras is homomorphically closed, we have, as usual, the following result.

Proposition 1.7. Let $\mathscr{W}$ be a universal variety of $\Omega$-algebras, containing all zeroalgebras. Then in $\mathscr{W}$ the lower radical class defined by the class of zeroalgebras contains all nilpotent algebras. 
For the free algebra $F$ we shall denote by $F^{n}$ the subalgebra (=T-ideal) generated by all words of length $\geqq n$.

Proposition 1.8. Let $\mathscr{W}$ be a universal variety of $\Omega$-algebras such that

and

(i) all zeroalgebras are in $\mathscr{W}^{-}$

(ii) $\bigcap_{n} F^{n}=0$.

Then any idempotent variety in $\mathscr{W}$ which contains all zeroalgebras must contain all of $\mathscr{W}$.

Proof. By Theorem 1.4 and Proposition 1.7, an idempotent variety $\mathscr{V}$ which contains the zeroalgebras must contain all nilpotent algebras and so, in particular, the algebras $F / F^{n}$ for each free algebra $F$. But $F$ is a subdirect product the $F / F^{n}$, so $\mathscr{V}$ contains all free algebras and hence all algebras.

CoROllary 1.9. If, in addition to the conditions in Proposition 1.8, $\Omega$ is a principal ideal domain, then any idempotent variety which contains a zeroalgebra $(\neq 0)$ contains all of $\mathscr{W}$.

Proof. We need only show that $\Omega^{0}$, the zeroalgebra on the additive module of $\Omega$, is in each idempotent variety $\mathscr{Y}$ containing a zeroalgebra $A \neq 0$, since then $\mathscr{V}$ contains all cyclic zeroalgebras and all homomorphic images of direct sums of these, i.e., all zeroalgebras. of must certainly contain a cyclic zeroalgebra $C$. If $C \nRightarrow \Omega^{\circ}$, then $C$ has as a homomorphic image the zeroalgebra on a cyclic module $\Omega /(\pi)$, where $\pi$ is prime. The latter module is in $\mathscr{Y}$, and arguing inductively from the exact sequences of modules

$$
0 \longrightarrow\left(\pi^{n}\right) /\left(\pi^{n+1}\right) \longrightarrow \Omega /\left(\pi^{n+1}\right) \longrightarrow \Omega /\left(\pi^{n}\right) \longrightarrow 0,
$$

observing that $\left(\pi^{n}\right) /\left(\pi^{n+1}\right) \cong \Omega /(\pi)$ for all $n$, we see that $\mathscr{Y}$ contains the zeroalgebra on $\Omega /\left(\pi^{n}\right)$ for all $n$. But $\Omega^{0}$ is a subdirect product of these.

The conditions of Proposition 1.8 are satisfied, for example, by the varieties of associative, alternative, Jordan, power-associative and arbitrary algebras. There are varieties $\mathscr{W}$ which contain all zeroalgebras but do not satisfy the requirement for free algebras: consider the variety of associative rings which are extensions of zerorings by boolean rings. In this case we have $F^{n}=F^{2} \neq 0$ for all $n \geqq 2$, since for any two generators $x, y$ of $F$ we have $\left(x^{n-1}+x\right)\left(y^{n-1}+y\right)=0$, whence $x y \in F^{n}$ and thus $F^{2} \subseteq F^{n}$ for all $n \geqq 2$.

The next result will enable us to specify some further universal varieties in which every idempotent variety has attainable identities. 
THEOREM 1.10. Let $\mathscr{V}$ be an idempotent variety, I the corresponding T-ideal. For every algebra $A, A(I, 1)$ is the ideal of $A$ generated by $A(I, 2)$.

Proof. Let $K$ be the ideal of $A$ generated by $A(I, 2)$. Then $K \triangleleft A(I, 1)$ and $A(I, 1) / K$ is a homomorphic image of $A(I, 1) / A(I, 2)$ and the latter is in $\mathscr{T}$, so $A(I, 1) / K$ is in $\mathscr{V}$. From the exact sequence

$$
0 \longrightarrow A(I, 1) / K \longrightarrow A / K \longrightarrow A / A(I, 1) \longrightarrow 0
$$

we see that $A / K$ is in $\mathscr{V}$, whence $(A, 1) \subseteq K$.

COROLLARY 1.11. If accessible (=subnormal) subalgebras are ideals in $\mathscr{W}$, then all idempotent varieties in $\mathscr{W}$ have attainable identities.

COROLlary 1.12. If $\Omega$ is a principal ideal domain and $\mathscr{W}$ satisfies the hypotheses of Proposition 1.8, then all idempotent varieties in $\mathscr{W}$ have attainable identities in the following situations:

(i.) cy्f is the variety of associative algebras;

(ii) $\mathscr{W}$ is the variety of alternative algebras;

(iii) $\mathscr{W}$ is the variety of Jordan algebras and $1 / 2 \in \Omega$.

Proof. We will show that in every case, we have:

If $C \triangleleft B \triangleleft A, B$ is the ideal of $A$ generated by $C$ and $C \neq B$, then $B / C$ contains a nonzero nilpotent element.

Then, when $\mathscr{V}$ is an idempotent variety with $T$-ideal $I$, it will follow from Theorem 1.10 that $A(I, 1) / A(I, 2)$ has nilpotent elements if it is nonzero. Since $A(I, 1) / A(I, 2)$ is in $\mathscr{T}$, either $A(I, 1)=A(I, 2)$ or $\mathscr{V}$ contains a zeroalgebra. In the latter case, $\mathscr{V}=\mathscr{W}$, by Corollary 1.9 , and certainly has attainable identities. If $\mathscr{V} \neq \mathscr{W}$, then $A(I, 1)=$ $A(I, 2)$ for all $A$, i.e. $\mathscr{V}$ has attainable identities.

The following observations establish (*):

(i) Andrunkievich's well-known result ([1], Lemma 4; see also [5], p. 107) says that $(B / C)^{3}=0$.

(ii) Hentzel and Slater [10] showed that $B / C$ is locally nilpotent for alternative rings. This is still true with a ring of operators.

(iii) Slin'ko [29] has shown that either $B / C$ contains a nilpotent ring-ideal or $C \triangleleft A$, i.e. $C=B$.

Under the conditions of Corollary 1.9, if $A$ belongs to an idempotent variety $\mathscr{V} \neq \mathscr{W}$, then no algebra in $\mathscr{V}$ can contain in nonzero nilpotent element and hence for every $a \in A$ we have $[a]=[a]^{2}$, where $[a]$ is the subalgebra generated by $a$. If in addition all algebras in 
$\mathscr{W}$ are power-associative, this means that $a=\sum_{i=2}^{k !} \alpha_{i} a^{i}$ for some $\alpha_{2}, \cdots, \alpha_{k} \in \Omega$. Under the additional restriction that $\Omega$ is the ring of integers or a finite prime field, this in turn means that $a=a^{n(a)}$ for some $n(a)>1$ (Osborn [21], p. 321).

For associative rings much of the material of this section has been covered. Armendariz [3] proved the relevant special case of Corollary 1.9 by using, inter alia, a result of Amitsur on free associative rings. Shevrin and Martynov [25] showed that the nontrivial varieties of associative rings with attainable identities are precisely the varieties generated by finite sets of finite fields, while Martynov [19] proved that these are precisely the nontrivial idempotent varieties. For a purely radical-theoretic account of semi-simple radical classes in this context see [31] and [9]. In particular, it is shown in [9] that the classes

$$
\mathscr{\mathscr { V }}_{n}=\left\{A \mid a^{n}=a \forall a \in A\right\}, \quad n=2,3,4, \cdots
$$

are semi-simple radical classes, though there are others. It has been widely accepted ([17], [30], [35], [36]) that the $\mathscr{V}_{n}$ are the only (nontrivial) semi-simple radical classes. By Theorem 1.5, the identities $x^{n}-x$ are all attainable for associative rings. It was asserted in [34] that $x^{2}-x$ is not attainable, and in [11] that only $x=x$ and $x=y$ are attainable.

We conclude this section with a couple of remarks about varieties in an arbitrary $\mathscr{W}$.

Following Leavitt [15], we say that for a class $\mathscr{M}$ of algebras which defines an upper radical class $U(\mathscr{C})$, the latter has the intersection property if

$$
U(\mathscr{C})(A)=\bigcap\{J \triangleleft A \mid A / J \in \mathscr{C}\}
$$

for all algebras $A$. If $\mathscr{V}$ is a variety with $T$-ideal $I$, then

$$
\bigcap\{J \triangleleft A \mid A / J \in \mathscr{Y}\}=A(I),
$$

so $\mathscr{V}$ has the intersection property if and only if $U(\mathscr{V})(A)=A(I)$ for all $A$. Then (cf. the proof of Theorem 1.5) we have

Proposition 1.13. For a variety $\mathscr{T}, U(\mathscr{V})$ has the intersection property if and only if $\mathscr{Y}$ has attainable identities or, equivalently, is a semi-simple class.

It follows from a result of Enerson and Leavitt ([6], Theorem 2) that varieties $\mathscr{A}, \mathscr{B}$ determine the same upper radical class if and only if every nonzero algebra in $\mathscr{A}$ (resp. $\mathscr{B}$ ) has a nonzero homomorphic image in $\mathscr{B}$ (resp. $\mathscr{A}$ ). 
As we have seen, the radical class $\mathscr{R}_{I}$ defined by a $T$-ideal $I$ need not be strict. On the other hand, for every strict radical class $\mathscr{R}, \mathscr{R}(F)$ is a $T$-ideal and so $\mathscr{R}$ determines a variety. It would be interesting to know the precise connection between $\mathscr{R}$ and this variety.

2. Associative results. In this section we shall work with associative algebras over a field $K$ and obtain results analogous to those obtained by Stewart [31] for associative rings. By Corollaries 1.9 and 1.12 , the nontrivial semi-simple radical classes (=idempotent varieties) contain no zeroalgebras and thus are subclasses of

$$
\mathscr{B}_{1}{ }^{K}=\left\{A \mid[a]=[a]^{2} \forall a \in A\right\} \text {. }
$$

Then as for rings ([2], Theorem 2; [31], Theorem 2.1), every algebra in $\mathscr{B}_{1}{ }^{K}$ is a subdirect product of algebras from $\mathscr{B}_{1}{ }^{K}$ without zerodivisors.

Proposition 2.1 (cf. [31], Lemma 3.2). A nonzero algebra $A \in \mathscr{B}_{1}{ }^{K}$ without zerodivisors is an algebraic division algebra.

Proof. If $0 \neq a \in A$, then $a \in[a]^{2}$, so $a=\sum_{i=2}^{k} \alpha_{i} a^{i}$ for suitable nonzero $\alpha_{2}, \cdots, a_{k} \in K$. Thus $A$ is algebraic over $K$. The rest of the proof follows as in [31].

Using Proposition 2.1 and arguing as in [31], Theorem 4.3 we obtain

Proposition 2.2. If $\mathscr{R}$ is a nontrivial semi-simple radical class, then $\mathscr{R}$ consists of all subdirect products of division algebras in $\mathscr{R}$.

THeORem 2.3. If $K$ is infinite, there are no nontrivial radical semi-simple classes of associative K-algebras.

Proof. Suppose $\mathscr{R}$ is such a class. Let $\mathscr{F}$ be the set of division algebras in $\mathscr{R}$. Since $\mathscr{R}$ is strongly hereditary, $K$ is in $\mathscr{F}$. Since $\mathscr{R}$ is a variety, it contains $\Pi K^{(n)}, n=1,2, \cdots$, where each $K^{(n)}=K$. Thus $\Pi K^{(n)} \in \mathscr{B}_{1}{ }^{K}$. If $u=\left(u_{1}, u_{2}, \cdots\right) \in \Pi K^{(n)}$, then $u=\sum_{i=2}^{k} \alpha_{i} u^{i}$ for some $\alpha_{2}, \cdots, \alpha_{k} \in K$, so each $u_{n}$ is a root of $\sum_{i=2}^{k} \alpha_{i} x^{i}-x$. But then $u_{n}$ can have only finitely many values. Hence there is no such $\mathscr{R}$.

Now consider finite fields $K$. By Theorem 2, p. 183 of [12], the division algebras of Proposition 2.1 are now commutative, and so are extension fields of $K$. We shall call them $K$-fields in the sequel. 
Theorem 2.4 (cf. [31], Theorem 3.4 and Corollary 3.5). The following conditions are equivalent for a K-algebra $A$.

(i) $A \in \mathscr{B}_{1}{ }^{K}$.

(ii) Every finitely generated subalgebra of $A$ is a finite direct sum of finite $K$-fields.

(iii) For every $a \in A$ there exists an integer $n(a)>1$ such that $a^{n(a)}=a$.

Proof. (i) $\Rightarrow$ (ii): Since $\mathscr{B}_{1}{ }^{K}$ is strongly hereditary, we need only look at finitely generated algebras $A \in \mathscr{B}_{1}{ }^{K}$. Being a subdirect product of $K$-fields (see Proposition 2.1 and the remark preceding it) $A$ is commutative. Since any generator $u$ of $A$ satisfies an equation

$$
u=\sum_{i=2}^{k} \alpha_{i} u^{i} ; \alpha_{2}, \cdots, \alpha_{k} \in K,
$$

it follows that $A$ is finite dimensional and hence finite. Also $A$ has no nilpotent elements, so $A$ is a finite direct sum of $K$-fields. Since $A$ is finite, so are the fields.

(ii) $\Rightarrow$ (iii): Each $a \in A$ is contained in a finite direct sum of finite fields, so a suitable $n(a)$ exists.

(iii) $\Rightarrow$ (i): This is clear.

Proposition 2.5 (cf. [31], Proposition 3.7). Let $\mathscr{F}$ be a finite, strongly hereditary set of finite $K$-fields. $A K$-algebra $A$ is a subdirect product of $K$-fields in $\mathscr{F}$ if and only if every finitely generated subalgebra of $A$ is a finite direct sum of $K$-fields from $\mathscr{F}$.

THEOREM 2.6. Let $K$ be a finite field. There is a bijection from the set of nonempty, finite, strongly hereditary sets of finite K-fields to the set of nontrivial semi-simple radical classes of associative $K$-algebras, given by

$$
\mathscr{F} \longmapsto\{A \mid A \text { is a subdirect product of } K \text {-fields in } \mathscr{F}\} \text {. }
$$

Proof. Let $\mathscr{R}$ be a nontrivial semi-simple radical class, $\mathscr{F}(\mathscr{R})$ the class of $K$-fields in $\mathscr{R}$. As in the proof of Theorem 4.3 of [31], it follows that $\mathscr{F}(\mathscr{R})$ is finite and strongly hereditary, all $K$-fields in $\mathscr{F}(\mathscr{R})$ are finite and $\mathscr{R}$ consists precisely of the subdirect products of $K$-fields from $\mathscr{F}(\mathscr{R})$. Conversely, if $\mathscr{F}$ is a finite, strongly hereditary set of finite $K$-fields, the class $\mathscr{C}(\mathscr{F})$ of subdirect products of $K$-fields from $\mathscr{F}$ is the semi-simple class defined by $\mathscr{F}$ ([32], Theorem 1; see also [5], p. 121) and hence is closed under extensions. But an algebra $A$ belongs to $\mathscr{C}(\mathscr{F})$ if and only if every finitely generated subalgebra of $A$ is a finite direct sum of $K$-fields from 
$\mathscr{F}$ (Proposition 2.5). From this it follows that $\mathscr{C}(\mathscr{F})$ is strongly hereditary and homomorphically closed. Thus $\mathscr{C}(\mathscr{F})$ is an idempotent variety, i.e. a semi-simple radical class. The correspondence is a bijection since it associates with each set of $K$-fields the semi-simple class which it generates.

3. Miscellaneous results. We conclude with a few examples, some of a rather negative character, involving idempotent varieties and semi-simple radical classes.

THeORem 3.1. In every power-associative universal variety $\mathscr{W}$, the variety

$$
\mathscr{V}_{2}=\left\{A \mid a^{2}=a \forall a \in A\right\}
$$

is idempotent.

Proof. All rings in $\mathscr{T}_{2}$ are commutative and have characteristic 2 , by the standard argument. If $I \triangleleft A$ and $I, A / I$ are in $\mathscr{\mathscr { T }} 2$, then for $a \in A$ we have $a^{2}-a \in I$ and thus $a^{2}-a=\left(a^{2}-a\right)^{2}=a^{4}-2 a^{3}+a^{2}$, i.e. $a^{4}+a-2 a^{3}=0$. Also $2\left(a^{2}-a\right)=0$, i.e. $2 a^{2}=2 a$, while $2 a$ is also in $I$, so that $2 \alpha=(2 a)^{2}=4 a^{2}=2.2 \alpha^{2}=2.2 \alpha$ and thus $2 a=0$. Hence $a^{4}+a=a^{4}-a=0$. But since $I \triangleleft A, a^{3}-a^{2}$ is also in $I$, so $a^{3}-a^{2}=a^{6}-a^{4}=a^{4} a^{2}-a^{4}=a a^{2}-a=a^{3}-a$, whence $a=a^{2}$. Thus $\mathscr{V}_{2}$ is closed under extensions.

When $\mathscr{W}$ is the class of associative rings, $\mathscr{V}_{2}$ is well known to be a semi-simple radical class (see [31]). Using Corollary 1.12, we get

COROLlaRy 3.2. The identity $x^{2}=x$ is attainable for alternative rings.

THEOREM 3.3. Let $\mathscr{Z}$ be a finite set of finite fields and simple Jordan rings of the kind described in Proposition 15.4 of [21], all having the same prime characteristic $p \neq 2$. Then the variety $\mathscr{V}$ of Jordan rings generated by $\mathscr{Z}$ has attainable identities.

Proof. For every $a \in A \in \mathscr{X}$, we have $a^{n(a)}=a$, for some $n(a)>1$ (see [21], Proposition 15.4). Since all the rings in $\mathscr{P}$ are finite we can find an $m>r$ such that $a^{m}=a$ for $a$ ll $a \in A \in \mathscr{X}$. Hence all rings in $\mathscr{V}$ satisfy $x^{m}=x$.

Let $\mathscr{P}$ be the class of Jordan rings $R$ such that for all $r \in R$ there exists $n(r)>1$ such that $r^{n(r)}=r$. If $I$ is an ideal of a Jordan ring $B$ such that $I$ and $B / I$ are in $\mathscr{P}$, then for $b \in B$, we have 
$b^{k}-b \in I$ for some $k>1$ and $b^{k}-b=\left(b^{k}-b\right)^{j}$ for some $j>1$. By Theorem 13.2 of [21], $B$ belongs to $\mathscr{P}$.

Now $\mathscr{V} \subseteq \mathscr{P}$. Let $J$ be an ideal of a Jordan ring $A$ such that $J$ and $A / J$ are in $\mathscr{V}$. Then $A$ is in $\mathscr{P}$, so by Theorem 15.11 of [21], $A$ is a subdirect product of simple rings. Let $L$ be an ideal of $A$ such that $A / L$ is one of these rings. Then $A /(J+L)$ is a homomorphic image of $A / L$, so either (i) $A /(J+L)=0$ or (ii) $A /(J+L)=A / L$. In case (i), we have $A=J+L$ so $A / L=(J+L) / L \cong J / J \cap L \in \mathscr{Y}$, while in case (ii), we have $A / L=A /(J+L) \cong[A / L] /[(J+L) / L]$ so $J+L=L$, i.e. $J \subseteq L$ and thus $A / L$, as a homomorphic image of $A / J$, is in $\mathscr{Y}$. Since $A$ is a subdirect product of rings in $\mathscr{Y}, A$ itself is in $\mathscr{Y}$.

Thus $\mathscr{V}$ is an idempotent variety consisting of rings of characteristic $p$, so $\mathscr{V}$ is an idempotent variety of Jordan algebras over the field $Z(p)$ of $p$ elements. By Corollary 1.12 (iii), $\mathscr{V}$ is a semisimple class of $Z(p)$-algebras. Let $S$ be a Jordan ring of which every nonzero ideal has a nonzero homomorphic image in $\mathscr{V}$ and suppose $p S \neq 0$. Then $p S \triangleleft S$, so $(p S) / T \in \mathscr{V}$ for some $T \triangleleft p S$. But then $(p S) / T$ has characteristic $p$, so that $p^{2} S \subseteq T$, which means that $(p S) / T$ is a zeroring, whereas $\mathscr{V}$ contains no ring with a nonzero nilpotent element. Thus $p S=0$ and every ideal of $S$ is a $Z(p)$-ideal. Since $\mathscr{V}$ is a semi-simple class of $Z(p)$-algebras, it follows that $S$ is in $\mathscr{V}$. Hence $\mathscr{V}$ is a semi-simple class of rings and so, by Theorem 1.5, has attainable identities.

We proceed now to some universal varieties in which there are no nontrivial varieties with attainable identities.

Firstly an example somewhat removed from what we have been looking at: Theorems 1.4 and 1.5 have exact analogues for groups (see also [24] in this connection); on the other hand, the Neumanns [20] and Shmel'kin [28] have shown that the set of nontrivial group varieties is the free semigroup (relative to $\circ$ ) generated by the indecomposable varieties, and as such has no idempotents. Thus there are no nontrivial attainable sets of identities for groups. This was also pointed out by Mal'tsev [18] and was established in another way by Tamura [33].

Recall that a Schreier variety of algebras over a field is one in which nonzero subalgebras of free algebras are free.

Proposition 3.4. If a universal variety $\mathscr{W}$ of algebras over a field is a Schreier variety, it has no nontrivial varieties with attainable identities. 
Proof. Let $F$ be a free algebra in $\mathscr{W}$ on $\aleph_{0}$ generators, $I$ a nonzero $T$-ideal $\neq F$. Then $I=F(I)$ is free. Let $\left\{e_{\lambda} \mid \lambda \in \Lambda\right\}$ be a set of free generators of $I$. We shall prove that $I(I)$ contains no $e_{\lambda}$, whence $I \neq I(I)=F(I, 2)$. Since $I \neq F$, there is a nonzero algebra $A$ in the variety defined by $I$. Suppose $e_{\mu}$ is in $I(I)$, for some $\mu \in \Lambda$, say $e_{\mu}=g\left(e_{\lambda_{1}}, \cdots, e_{\lambda_{n}}\right)$ for some $g \in I$ and $\lambda_{1}, \cdots, \lambda_{n} \in A$. Choose $a$ $(\neq 0)$ in $A$ and define $\phi: I \rightarrow A$ by $\phi\left(e_{\mu}\right)=a$ and $\phi\left(e_{\lambda}\right)=0$ for $\lambda \neq \mu$. Then we have the contradiction $0 \neq a=\phi\left(e_{\mu}\right)=g\left(\phi\left(e_{\lambda_{1}}\right), \cdots, \phi\left(e_{\lambda_{n}}\right)\right)=0$.

For any variety $\mathscr{V}$ of rings we shall denote by $\mathscr{V}_{K}$ the variety of algebras over a field $K$ whose underlying rings are in $\mathscr{V}$.

THEOREM 3.5. Let $\mathscr{W}$ be a universal variety of rings which contains all zerorings. If $\mathscr{W}_{K}$ has no nontrivial varieties with attainable identities for each field $K$, then $\mathscr{W}$ has none.

Proof. Suppose $\mathscr{W}_{K}$ has no nontrivial attainable identities for every field $K$, and let $\mathscr{O}$ be a variety in $\mathscr{W}$ with attainable identities. In what follows, $Q$ denotes the field of rational numbers. Let $A$ be a $\mathscr{W}_{Q}$-algebra, every nonzero ideal of which has a nonzero homomorphic image in $\mathscr{V}_{0}$. Then as a ring, $A$ has the property that every nonzero divisible ideal is a $Q$-algebra ideal and so has as a homomorphic image a nonzero algebra in $\mathscr{V}$, i.e., $A$ has a nonzero torsion-free homomorphic image in $\mathscr{T}$. If $I$ is an arbitrary nonzero ideal of $A$ (qua ring) then $I^{*}=\{a \in A \mid \exists n \neq 0$ such that $n a \in I\}$ is a nonzero divisible ideal, so, since $A$ is a $Q$-algebra, $I^{*} / K \in \mathscr{V}$ for some $K \triangleleft I^{*}$ such that $I^{*} / K$ is nonzero and torsion-free. Under these conditions, $I \nsubseteq K$, so $I / I \cap K \cong(I+K) / K$ is a nonzero homomorphic image of $I$ which belongs to $\mathscr{T}$. Since $\mathscr{V}$ is a semi-simple class, it follows that $A$ belongs to $\mathscr{V}$ and hence to $\mathscr{V}_{Q}$. But then $\mathscr{V}_{Q}$ is a semi-simple class in $\mathscr{W}_{Q}$, i.e., it has attainable identities there.

By assumption, $\mathscr{V}_{Q}=\{0\}$ or $\mathscr{W}_{Q}$ (Note that $\mathscr{W}_{Q} \neq\{0\}$.) In the

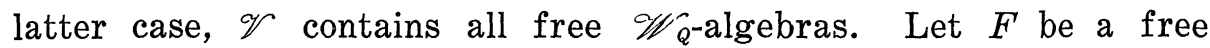
$\mathscr{W}$-ring of rank $\aleph_{0}$. Then by Theorem 4.4, p. 195 of [21], $Q \otimes_{Z} F$ or, equivalently, the minimal divisible extension $D(F / t(F))$ of $F / t(F)$ ([7] Theorem 119.1) where $t(F)$ is the torsion ideal of $F$, satisfies the identities of $\mathscr{W}$ and so is free of rank $\mathcal{W}_{0}$ in $\mathscr{W}_{Q}$. Then $\mathscr{V}$ contains $D\left(F / t\left(F^{\prime}\right)\right)$ and hence $F / t\left(F^{\prime}\right)$. But then $\mathscr{V}$ contains all torsion-free $\mathscr{W}$-rings. In particular, $\mathscr{V}$ contains the zeroring $Z^{\circ}$ on $Z$ and hence the zeroring $Z(p)^{0}$ on the cyclic group of order $p$, for all primes $p$.

For convenience, we shall denote by $\mathscr{C}_{p}$ the variety of algebras over the field $Z(p)$ of $p$ elements, which belong to $\mathscr{q}$, and so on. The above argument shows that $\mathscr{V}_{p} \neq\{0\}$ for all primes $p$. Let $B$ be a $\mathscr{W}_{p}$-algebra of which every nonzero algebra ideal has a nonzero homomorphic image in $\mathscr{T}_{p}$. Since every ring ideal of $B$ is an algebra 
ideal and $\mathscr{V}$ is a semi-simple class in $\mathscr{W}$, it follows that $B$ is in $\mathscr{T}$, whence $\mathscr{\mathscr { V }}_{p}$ is a semi-simple class in $\mathscr{W}_{p}$. Consequently (since $\mathscr{V}_{p}$ has attainable identities and $\left.\mathscr{\mathscr { V }}_{p} \neq\{0\}\right) \mathscr{\mathscr { V }}_{p}=\mathscr{V} \cap \mathscr{W}_{p}=\mathscr{W}_{p}$. Thus $\mathscr{V}$ contains all $\mathscr{W}$-rings $R$ for which $p R=0$.

Let $S$ be a $\mathscr{W}$-ring whose additive group is a $p$-group, and for each $n$ let $S\left[p^{n}\right]=\left\{a \in S \mid p^{n} a=0\right\}$. Then $S\left[p^{n+1}\right] / S\left[p^{n}\right] \in \mathscr{V}$ for all $n$, so by induction on $n$, each $S\left[p^{n}\right]$ is in $\mathscr{V}$. But then $S=\bigcup_{n} S\left[p^{n}\right] \in \mathscr{V}$. It follows that $\mathscr{Y}$ contains all torsion $\mathscr{W}$-rings, in particular $t(F)$, for the free $\mathscr{W}$-ring $F$. Since, as we've seen, $F / t(F)$ is in $\mathscr{Y}$, so is $F$, and thus $\mathscr{V}=\mathscr{W}$ if $\mathscr{V}_{Q} \neq\{0\}$.

Thus we may assume that $\mathscr{V}_{Q}=\{0\}$. As shown above, $\mathscr{V}_{p}$ is always a semi-simple radical class in $\mathscr{W}_{p}$ and so $\mathscr{\mathscr { N }}_{p}=\mathscr{W}_{p}$ or $\{0\}$. If $\mathscr{V}_{p}=\mathscr{W}_{p}$ for some $p$, then $\mathscr{V}$ contains the zeroring on $Z(p)$ and hence (cf. the proof of Corollary 1.9) all zerorings, including the torsionfree divisible ones, contradicting our assumption that $\mathscr{V}_{Q}=\{0\}$. Thus $\mathscr{T}_{p}=\{0\}$ for all primes $p$. If now $R$ is any ring in $\mathscr{V}$, then $R / p R=0$ and $R[p]=0$ for all primes $p$. This means that $R$ is torsion-free and divisible, so $R=0$. This completes the proof.

Combining Proposition 3.4 with Theorem 3.5, we get

COROLlaRY 3.6. Let $\mathscr{W}$ be a universal variety of rings, containing the zerorings, such that $\mathscr{W}_{K}$ is a Schreier variety for all fields $K$. Then $\mathscr{W}$ has no nontrivial varieties with attainable identities.

The Schreier varieties over an arbitrary field include the varieties of all algebras (Kurosh [13]), Lie algebras (Shirshov [26], Witt [37]), commutative and anticommutative algebras (Shirshov [27]). In the case of characteristic 0, Parfenov [22] obtained, for Lie algebras, the exact analogue of the group-theoretic result of the Neumanns and Shmel'kin quoted above, so there are no nontrivial idempotent varieties there. As we have remarked, we do not know of an example (anywhere) of an idempotent variety which does not have attainable identities, but it seems worth recording that an argument similar to that used for Theorem 3.5 establishes the following result.

THEOREM 3.7. Let $\mathscr{Y}$ be a universal variety of rings, containing all zerorings. If $\mathscr{W}_{K}$ has no idempotent varieties for each field $K$, then $\mathscr{W}$ has none.

\section{REFERENCES}

1. V. A. Andrunakievich, Radikaly assotsiativnykh kolets $I$, Mat. Sb., 44 (1958), 179212. (Translation: V. A. Andrunakievič Radicals of associative rings I, Amer. Math. Soc. Transl., (2) 52 (95-128)). 
2. V. A. Andrunakievich and Yu. M. Ryabukhin, Kol'tsa bez nil'potentykh elementov $i$ vpolne prosty, idealy, Dokl. Akad. Nauk. SSSR, 180 (1968), 9-11. (Translation: V. A. Andrunakievič and Ju. M. Rjabuhin, Rings without nilpotent elements and completely simple ideals, Soviet Math. Dokl., 9 (1968), 565-568).

3. E. P. Armendariz, Closure properties in radical theory, Pacific J. Math., 26 (1968), $1-7$.

4. P. M. Cohn, Universal Algebra, Harper and Row, New York, 1965.

5. N. J. Divinsky, Rings and Radicals, Allen and Unwin, London, 1965.

6. P. O. Enerson and W. G. Leavitt, The upper radical construction, Publ. Math. Debrecen, 20 (1973), 219-222.

7. L. Fuchs, Infinite Abelian Groups, Vol. II, Academic Press, New York and London, 1973.

8. B. J. Gardner, Some radical constructions for associative rings, J. Austral. Math. Soc., 18 (1974), 442-446.

9. B. J. Gardner and P. N. Stewart, On semi-simple radical classes, Bull. Austral. Math. Soc., 13 (1975), 349-353.

10. I. R. Hentzel and M. Slater, On the Andrunakievich lemma for alternative rings, J. Algebra, 27 (1973), 243-256.

11. A. A. Iskander, Product of ring varieties and attainability, Trans. Amer. Math. Soc., 193 (1974), 231-238.

12. N. Jacobson, Structure of rings, Amer. Math. Soc. Colloquium Publ. XXXVII, Providence, 1968.

13. A. G. Kurosh, Neassotsiativnye svobodnye algebry $i$ svobodnye proizvedeniya algebr, Mat. Sb. (Recueil Mathématique), 20 (1947), 239-262.

14. W. G. Leavitt, The general theory of radicals, Mimeographed notes, University of Nebraska, Lincoln (n.d.).

15. - The intersection property of an upper radical, Arch. Math., (Basel), 24 (1973), 486-492.

16. W. G. Leavitt and E. P. Armendariz, Nonhereditary semisimple classes, Proc. Amer. Math. Soc., 18 (1967), 1114-1117.

17. L. C. A. van Leeuwen and T. L. Jenkins, A note on radical semisimple classes, Publ. Math. Debrecen, 21 (1974), 179-184.

18. A. I. Mal'tsev, Ob umnozhenii klassov algebraicheskikh sistem, Sibirskii Mat. Zhurnal, 8 (1967), 346-365.

19. L. M. Martynov, O razreshimykh kol'tsakh, Ural. Gos. Univ. Mat. Zap. 8, Tetrad' 3 (1972), 82-93.

20. B. H. Neumann, H. Neumann and P. M. Neumann, Wreath products and varieties of groups, Math. Z., 80 (1962), 44-62.

21. J. M. Osborn, Varieties of algebras, Advances in Math., 8 (1972), 163-369.

22. V. A. Parfenov, O mnogoobraziyakh algebr Li, Algebra i Logika 6, No. 4 (1967), 61-73.

23. C. Procesi, Rings with polynomial identities, Marcel Dekker, New York, 1973.

24. K. K. Shchukin, O verbantnykh radikalakh grupp, Kishinevskii Gos. Univ. Uchenye Zapiski, 82 (1965), 97-99.

25. L. N. Shevrin and L. M. Martynov, O dostizhimykh klassakh algebr, Sibirskii Mat. Zhurnal, 12 (1971), 1363-1381.

26. A. I. Shirshov, Podalyebry svobodnykh Lievykh algebr, Mat. Sb., 33 (1953), 441452.

27. A. I. Shirshov, Podalgebry svobodnykh kommutativnykh $i$ svobodnykh antikommutativnykh algebr, Mat. Sb., 34 (1954), 81-88.

28. A. L. Shmel'kin, Polugruppa mnogoobrazii grupp, Dokl. Akad. Nauk. SSSR, 149 (1963), 543-545. (Translation: A. L. Šmel'kin, The semigroup of group manifolds, Soviet Math. Dokl., 4 (1963), 449-451.

29. A. M. Slin'ko, O radikalakh Iordanovkh kolets, Algebra i Logika 11, No. 2 (1972), 
206-215.

30. R. L. Snider, Complemented hereditary radicals, Bull. Austral. Math. Soc., 4 (1971), 307-320.

31. P. N. Stewart, Semi-simple radical classes, Pacific J. Math., 32 (1970), 249-254. 32. A Suliński, Nekotorye voprosy obshchei teorii radikalov, Mat. Sb., 44 (1958), 273286.

33. T. Tamura, Attainability of systems of identities on semigroups, J. Algebra, 3 (1966), 261-276.

34. T. Tamura and F. M. Yaqub, Examples related to attainability of identities on lattices and rings, Math. Japon., 10 (1965), 35-39.

35. R. Wiegandt, Radical and semisimple classes of rings, Queen's Papers in Pure and Applied Mathematics, No. 37, Kingston, 1974.

36. R. Wiegandt, Homomorphically closed semisimple classes, Studia Univ. Babeș-Bolyai Ser. Math. Mech., 2 (1972), 17-20.

37. E. Witt, Die Unterringe der freien Lieschen Ringe, Math. Z., 64 (1956), 195-216.

Received June 24, 1975 and in revised form October 21, 1975. The author thanks the referee for detecting an omission in an earlier version of the proof of Theorem 3.5 and for other useful comments. Some of the results presented here were obtained during a visit by the author to the Department of Mathematics, Institute of Advanced Studies, Australian National University, Canberra. The assistance and facilities provided by that department are gratefully acknowledged.

University of Tasmania, Hobart, Australia 


\section{PACIFIC JOURNAL OF MATHEMATICS}

\section{EDITORS}

RICHARD ARENS (Managing Editor)

University of California

Los Angeles, California 90024

\author{
R. A. Beaumont \\ University of Washington \\ Seattle, Washington 98105
}

\section{J. DugundjI}

Department of Mathematics University of Southern California Los Angeles, California 90007

D. Gilbarg and J. Milgram

Stanford University

Stanford, California 94305

\section{ASSOCIATE EDITORS}
E. F. BECKENBACH
B. H. NeumanN
F. WOLF
K. YoshidA

\section{SUPPORTING INSTITUTIONS}

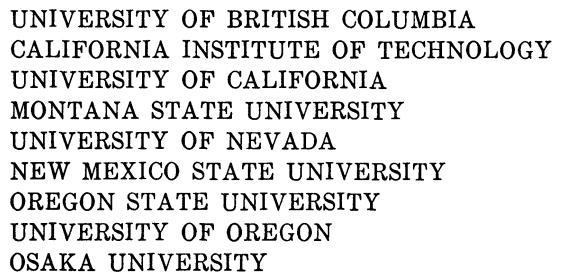

UNIVERSITY OF BRITISH COLUMBIA CALIFORNIA INSTITUTE OF TECHNOLOGY UNIVERSITY OF CALIFORNIA MONTANA STATE UNIVERSITY UNIVERSITY OF NEVADA NEW MEXICO STATE UNIVERSITY OSAKA UNIVERSITY OREGON STATE UNIVERSITY UNIVERSITY OF OREGON

\author{
UNIVERSITY OF SOUTHERN CALIFORNIA \\ STANFORD UNIVERSITY \\ UNIVERSITY OF TOKYO \\ UNIVERSITY OF UTAH \\ WASHINGTON STATE UNIVERSITY \\ UNIVERSITY OF WASHINGTON \\ AMERICAN MATHEMATICAL SOCIETY
}

The Supporting Institutions listed above contribute to the cost of publication of this Journal, but they are not owners or publishers and have no responsibility for its content or policies.

Mathematical papers intended for publication in the Pacific Journal of Mathematics should be in typed form or offset-reproduced, (not dittoed), double spaced with large margins. Please do not use built up fractions in the text of your manuscript. You may however, use them in the displayed equations. Underline Greek letters in red, German in green, and script in blue. The first paragraph or two must be capable of being used separately as a synopsis of the entire paper. Items of the bibliography should not be cited there unless absolutely necessary, in which case they must be identified by author and Journal, rather than by item number. Manuscripts, in triplicate, may be sent to any one of the editors. Please classify according to the scheme of Math. Reviews, Index to Vol. 39. All other communications should be addressed to the managing editor, or Elaine Barth, University of California, Los Angeles, California, 90024.

The Pacific Journal of Mathematics expects the author's institution to pay page charges, and reserves the right to delay publication for nonpayment of charges in case of financial emergency.

100 reprints are provided free for each article, only if page charges have been substantially paid. Additional copies may be obtained at cost in multiples of 50 .

The Pacific Journal of Mathematics is issued monthly as of January 1966. Regular subscription rate: $\$ 72.00$ a year $(6$ Vols., 12 issues). Special rate: $\$ 36.00$ a year to individual members of supporting institutions.

Subscriptions, orders for back numbers, and changes of address should be sent to Pacific Journal of Mathematics, 103 Highland Boulevard, Berkeley, California, 94708.

PUBLISHED BY PACIFIC JOURNAL OF MATHEMATICS, A NON-PROFIT CORPORATION

Printed at Kokusai Bunken Insatsusha (International Academic Printing Co., Ltd.), 8-8, 3-chome, Takadanobaba, Shinjuku-ku, Tokyo 160, Japan. 


\section{Pacific Journal of Mathematics}

\section{Vol. 61, No. 2 December, 1975}

Graham Donald Allen, Francis Joseph Narcowich and James Patrick Williams, An operator version of a theorem of Kolmogorov .......................

Joel Hilary Anderson and Ciprian Foias, Properties which normal operators share with normal derivations and related operators . . . . . . . . . . . . . . . . . . . . .

Constantin Gelu Apostol and Norberto Salinas, Nilpotent approximations and

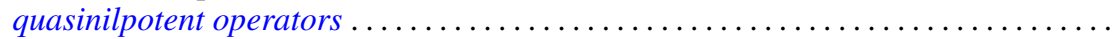

James M. Briggs, Jr., Finitely generated ideals in regular $F$-algebras . . . . . . . . . . .

Frank Benjamin Cannonito and Ronald Wallace Gatterdam, The word problem and power problem in 1-relator groups are primitive recursive ..................

Clifton Earle Corzatt, Permutation polynomials over the rational numbers ...........

L. S. Dube, An inversion of the $S_{2}$ transform for generalized functions . . . . . . . . . . William Richard Emerson, Averaging strongly subadditive set functions in unimodular

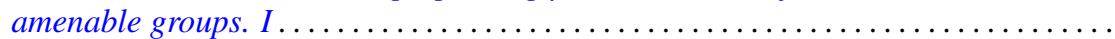

Barry J. Gardner, Semi-simple radical classes of algebras and attainability of

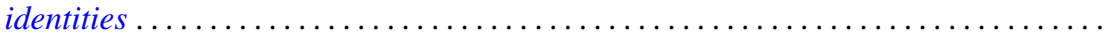

Irving Leonard Glicksberg, Removable discontinuities of A-holomorphic functions ....

Fred Halpern, Transfer theorems for topological structures . . . . . . . . . . . . . . .

H. B. Hamilton, T. E. Nordahl and Takayuki Tamura, Commutative cancellative

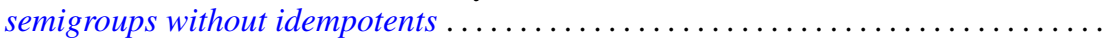

Melvin Hochster, An obstruction to lifting cyclic modules .....................

Alistair H. Lachlan, Theories with a finite number of models in an uncountable power

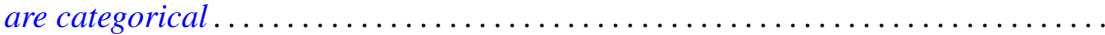

Kjeld Laursen, Continuity of linear maps from $C^{*}$-algebras . . . . . . . . . . . . .

Tsai Sheng Liu, Oscillation of even order differential equations with deviating arguments ....

Jorge Martinez, Doubling chains, singular elements and hyper- $Z$

Mehdi Radjabalipour and Heydar Radjavi, On the geometry of num Thomas I. Seidman, The solution of singular equations, I. Linear equations in Hilbert

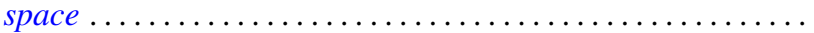

R. James Tomkins, Properties of martingale-like sequences ......

Alfons Van Daele, A Radon Nikodým theorem for weights on von Neumann

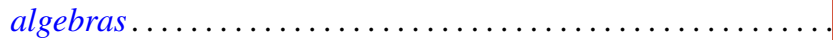

Kenneth S. Williams, On Euler's criterion for quintic nonresidues

Scott Andrew Wolpert, Noncompleteness of the Weil-Petersson metric for Teichmüller

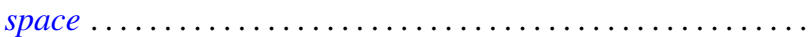

Volker Wrobel, Some generalizations of Schauder's theorem in locally convex

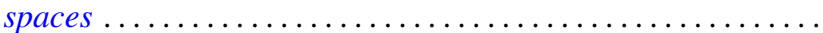

Kelly Denis McKennon, Corrections to: "Multipliers of type $(p, p)$ "; "Multipliers of type $(p, p)$ and multipliers of the group $L_{p}$-algebras"; "Multipliers and the

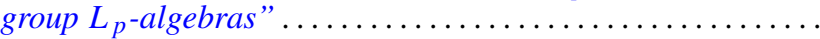

Andrew M. W. Glass, W. Charles (Wilbur) Holland Jr. and Stephen H. McCleary, Correction to: " $a *$-closures to completely distributive lattice-ordered

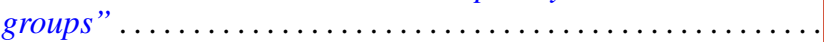

Zvi Arad and George Isaac Glauberman, Correction to: "A characteristic subgroup of

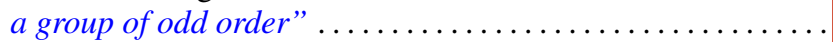

Roger W. Barnard and John Lawson Lewis, Correction to: "Subordination theorems

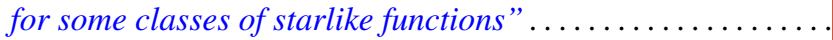

